\title{
New Condition of Automatic Continuity of Dense Range Homomorphisms on Jordan - Banach Algebras
}

\section{RUQAYAH N. BALO}

Department of Mathematics

College of Education

University of Mosul
NADIA A. ABDULRAZAQ

Department of Mathematics

College of Education

University of Mosul

\section{DUAA F. ABDULLAH}

Department of Mathematics

College of Education

University of Mosul

Received

23/12/2012
Accepted

06/03/2013

الملخص

المسألة المفتوحة التالية تتص على انه ، اذا كان

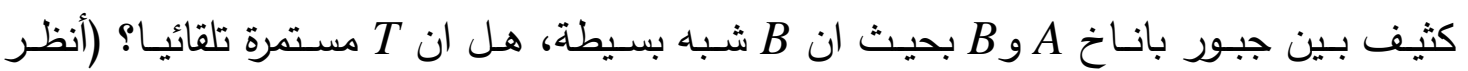

في [3] اعطي حلا جزئيا للمسألة اعلاه كالآتي:-

ليكن A و B جبور فريجيت بحيث ان B شبه بسيطة، نصف القطر الطيفي

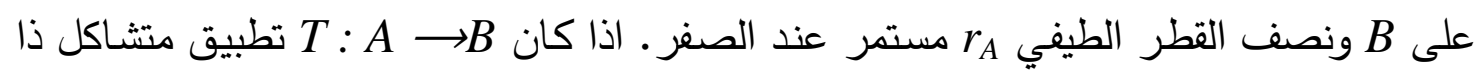
مستقر كثيف ، عندئذ T مستمرة تلقائيا. في هذا البحث برهنا النتيجة التالية: اذا كان B بحيث ان B $B^{+}$ الطيفي 


\section{ABSTRACT}

The following open problem stated that, if $T: A \rightarrow B$ is a dense range homomorphism between Banach algebras $A$ and $B$ such that $B$ is semisimple. Is $T$ automatically continuous? (see [1]).

In [3] given a partial solution of the above problem as follows:

Let $A$ and $B$ be Fréchet algebras such that $B$ is semi simple, the spectral radius $r_{B}$ is continuous on $B$ and the spectral radius $r_{A}$ is continuous at zero. If $T: A \rightarrow B$ is a dense range homomorphism, then $T$ is automatically continuous.

In this paper, we prove the following result :

If $T: A^{+} \rightarrow B^{+}$is a dense range homomorphism between Jordan Banach algebras $A^{+}$and $B^{+}$such that $B^{+}$is semi simple, the spectral radius $r_{B}+$ is continuous on $B^{+}$and the spectral $r_{A}+$ is continuous at zero, then $T$ is automatically continuous.

\section{Introduction :}

If $A$ and $B$ are Banach algebras, $B$ is semi simple and $T: A \rightarrow B$ is a dense range homomorphism, then the continuity of $\mathrm{T}$ is a long - standing open problem.

This is perhaps the most interesting open problem remains in automatic continuity theory for Banach algebras. (see [1]).

We recall that from [2], the radical of an algebra $A$, denoted by $\operatorname{rad} A$, is the intersection of all maximal left (right) ideals in $A$. The algebra $A$ is called semi simple if $\operatorname{rad} A=\{0\}$. In [3], for the algebra $A$ the spectrum of an element $x \in A$ is the set of all $\lambda \in \mathbb{C}$ such that $\lambda 1-x$ is not invertible in $A$ and is denoted by $\operatorname{Sp}(x)$ (or by $\operatorname{SpA}(x)$ ). Thus

$$
\operatorname{Sp}(x)=\{\lambda \in \mathbb{C}: \lambda 1-x \notin \operatorname{Inv}(A)\} .
$$

Also let $A$ be Banach algebra, then the spectral radius of $x$ (with respect to $A$ ) is denoted by $r(x)$ (or $r_{A}(x)$ ) and is defined by the formula

It is known that for any algebra $A$ we have

$$
r(x)=\operatorname{Sup}\{|\lambda|: \lambda \in \operatorname{Sp}(x)\} .
$$

$$
\operatorname{rad} A=\left\{x \in A: r_{A}(x y)=0 \text { for every } \mathrm{y} \in A\right\} .
$$

From [6], for $X, Y$ normed spaces and $T$ a linear mapping from $X$ into $Y$, then the separating subspace $S(T)$ of $T$ is defined as follows :

$S(T)=\left\{y \in Y: \exists\left\{x_{n}\right\} \subseteq X, x_{n} \rightarrow 0, T x_{n} \rightarrow y, \forall n \in N\right\}$.

We recall that a complex Jordan algebra $A$ is a non - associative and the product satisfies the identities $a b=b$ a and $(a b) a^{2}=\mathrm{a}\left(b a^{2}\right)$, for all $\mathrm{a}, \mathrm{b}$ in $A$. A unital Jordan - Banach algebra is a Jordan algebra with a complete norm satisfying $\|x y\| \leq\|x\|\|y\|$, for $x, y \in A$, and $\|1\|=1$. (see [4] ). The well - known example of Jordan - Banach 
algebra is that if we take any Banach algebra $A$, then $A^{+}$is a Jordan Banach algebra with a product defined as follows :

$$
\mathrm{a} \cdot \mathrm{b}=\frac{1}{2}(\mathrm{ab}+\mathrm{ba}) \quad \forall \mathrm{a}, \mathrm{b} \in A
$$

So $\left(A^{+},.\right)$is Jordan - Banach algebra over a field $\mathrm{F}$ of characteri stic $\neq 2$. (see $[5])$.

In this paper, we prove that :

Let $A^{+}$and $B^{+}$be Jordan - Banach algebras such that $B^{+}$is semisimple, the spectral radius $r_{B}+$ is continuous on $B^{+}$and the spectral radius $r_{A}+$ is continuous at zero. If $\mathrm{T}: A^{+} \rightarrow B^{+}$is a dense range homomorphism, then $T$ is automatically continuous.

This is in fact an extension of the open problem from the associative case to the more general situation of Jordan - Banach algebras.

\section{Fundamental Results :}

In this section we prove our fundamental following results

\section{Theorem 2.1 :}

Let $A^{+}$and $B^{+}$be Jordan - Banach algebras and $T: A^{+} \rightarrow B^{+}$a dense range homomorphism. Then the separating subspace $S(T)$ is a closed ideal of $B^{+}$.

\section{Proof:}

Clearly $S(T)$ is a closed linear subspace of $B+$. Let $y \in S(T)$ and $z \in B^{+}$. There exists a sequence $\left\{x_{n}\right\}$ in $A^{+}$such that $x_{n} \rightarrow 0$ and $T x_{n} \rightarrow y$. Moreover, $z=T x$ for some $x \in A^{+}$. Hence if $x x_{n}=x_{n} x$ then $x$. $x_{n} \rightarrow 0$ imply that $\frac{1}{2}\left(x x_{n}+x_{n} x\right) \rightarrow 0$ and this imply that $x x_{n} \rightarrow 0$

$$
\begin{aligned}
& \text { and } T\left(x . x_{n}\right)=\frac{1}{2} T\left(x x_{n}+x_{n} x\right) . \\
& \quad=\frac{1}{2}\left(T x T x_{n}+T x_{n} T x\right) . \\
& \quad=T x T x_{n} \rightarrow z y \text { and so } z y \in S(T)
\end{aligned}
$$

Similarly $y z \in S(T)$. Therefore, $S(T)$ is an ideal in $B^{+}$.

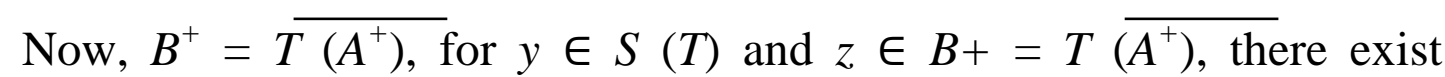
sequences $\left\{x_{n}\right\}$ in $A^{+}$and $\left\{z_{n}\right\}$ in $T\left(A^{+}\right)$such that $x_{n} \rightarrow 0$ in $A^{+}$, $z_{n} \rightarrow z$ and $T x_{n} \rightarrow y$ in $B^{+}$. Since $y z_{n}, z_{n} y \in S(T)$ and $y z_{n} \rightarrow y z, z_{n} y \rightarrow z y$ it follows that $y \overline{z, z y} \in S(T)=S(T)$.

\section{Theorem 2.2 :}

Let $A^{+}$and $B^{+}$be Jordan - Banach algebras such that $B^{+}$is semisimple, the spectral radius $r_{B^{+}}$is continuous on $B+$ and the spectral 
radius $r_{A}+$ is continuous at zero. If $T: A^{+} \rightarrow B^{+}$is a dense range homomorphism, then $T$ is automatically continuous.

\section{Proof:}

If $x y=y x$ for all $x$ and $y$ in $A^{+}$then

$$
\begin{aligned}
T(x . y)=T(y \cdot x) & =\frac{1}{2} T(x y+y x) \\
& =\frac{1}{2}(T x T y+T y T x) \\
& =T x T y \\
& =T y T x
\end{aligned}
$$

If $x$ is an arbitrary quasi - Invertible element of $A^{+}$, then there exists an element $y$ in $A^{+}$such that $x y=y x=x+y$. It follows that

$$
T x T y=T y T x=T x+T y
$$

That is $T y$ is Quasi - invertible element of $T x$. Hence, $T$ reduces the spectrum of elements. so,

$$
r_{B}+(T x) r_{A}+(x)
$$

For every $y \in S(T)$ there exists a sequence $\left\{x_{n}\right\}$ in $A^{+}$such that $x_{n} \rightarrow 0$ in $A^{+}$and $T x_{n} \rightarrow y$ in $B^{+}$. Since $r_{B}+(T x) \leq r_{A}+(x)$ for every $x \in A^{+}$ and $r_{A}+$ is continuous by assumption, we have $r_{A}+\left(x_{n}\right) \rightarrow 0$, then $r_{B}+\left(T x_{n}\right) \rightarrow 0$. On the other hand, again by continuity of $r_{B}+$ we have $r_{B}+\left(T x_{n}\right) \rightarrow r_{B}+(y)$. Hence

$$
r_{B}+(y)=0
$$

Since $T: A^{+} \rightarrow B^{+}$is a dense range homomorphism, by Theorem (2.1) $S(T)$ is an ideal in $B+$. Thus for every $z \in B^{+}, y z \in S(T)$. By (1) we get $r_{B}+(y z)=0$.

Since $\operatorname{rad} B^{+}=\left\{y \in B^{+}: r_{B}+(y z)=0\right.$ for every $\left.z \in B^{+}\right\}$, therefore $y \in \operatorname{rad} B^{+}$. So $S(T) \subseteq \operatorname{rad} B^{+}$. Since $B^{+}$is semi-simple, we have $S(T)=\{0\}$ and so $T$ is continuous by the closed graph theorem.

\section{Acknowledgement}

I would like to thank the referees for valuable conversations and comments. 


\section{REFERENCES}

[1] Bachar, J. M., Radical Banach Algebras and Automatic Continuity, Lecture Notes in Mathematics 975, Springer Verlag, Berlin Heidelberg New York, 1983.

[2] Dales, H. G., Banach Algebras and Automatic Continuity, London Mathematics Society Monographs 24, Clarendon Press, Oxford, 2000.

[3] Honary.T.GH., Automatic Continuity of Homomorphisms Between Banach Algebras and Fréchet Algebras,Bull. Iranian Math.Soc., 32 (2006), No.2, PP. 1-11.

[4] Maouche, A., Spectrum Preserving Linear Mappings For Scattered Jordan - Banach Algebras, Proc. Amer. Math. Soc., 127(1999), NO.11, PP.3187 - 3190 .

[5] Schafer, R. D., An Introduction to Non - Associative Algebras, Massachusetts Institute of Technology, Renewed 1994.

[6] Sinclair, A. M., Automatic Continuity of Linear Operators, London Mathematical Society, Lecture Note Series 21, Cambridge University Press, Cambredge, 1976. 